\title{
Predicting Acute Kidney Injury in Intensive Care Unit Patients: The Role of Tissue Inhibitor of Metalloproteinases-2 and Insulin-Like Growth Factor-Binding Protein-7 Biomarkers
}

\author{
Laura Di Leo $^{a}$ Federico Nalesso $^{b}$ Francesco Garzotto $^{a, b}$ Yun Xie ${ }^{a, c}$ \\ Bo Yang ${ }^{a, d}$ Grazia Maria Virzì ${ }^{a, b}$ Alberto Passannante ${ }^{a, e}$ Raffaele Bonato ${ }^{f}$ \\ Mariarosa Carta ${ }^{g}$ Davide Giavarina ${ }^{g}$ Dario Gregori ${ }^{\text {h }}$ Alessandra Brendolan ${ }^{a}$ b \\ Fiorenza Ferrari ${ }^{a} \quad$ Claudio Ronco ${ }^{a}$, b

\begin{abstract}
${ }^{a}$ International Renal Research Institute of Vicenza, San Bortolo Hospital, Vicenza, Italy; ${ }^{b}$ Department of Nephrology, Dialysis and Transplantation, San Bortolo Hospital, Vicenza, Italy; ' Department of Nephrology, Xin Hua Hospital Affiliated to Shanghai Jiaotong University School of Medicine, Shanghai, China; ${ }^{\text {d}}$ Department of Nephrology, First

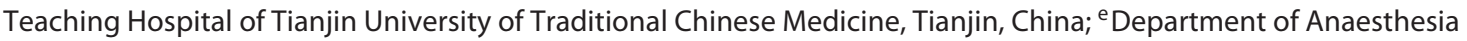
and Intensive Care, University of Trieste, Trieste, Italy; ${ }^{f}$ Department of Intensive Care, San Bortolo Hospital, Vicenza,

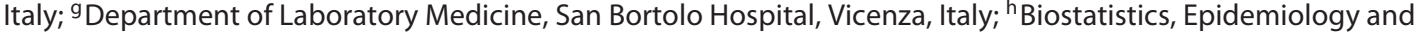
Public Health Unit, Department of Cardiac, Thoracic and Vascular Sciences, University of Padova, Padova, Italy
\end{abstract}

\section{Keywords}

Tissue inhibitor of metalloproteinases-2 and insulinlike growth factor-binding protein 7 . Cell cycle arrest biomarker · Acute kidney injury

\begin{abstract}
Background: Acute kidney injury (AKI) diagnosis is based on a rise in serum creatinine and/or fall in urine output. It has been shown that there are patients that fulfill AKI definition but do not have $\mathrm{AKI}$, and there are also patients with evidence of renal injury who do not meet any criteria for AKI. Recently the innovative and emerging proteomic technology has enabled the identification of novel biomarkers that allow improved risk stratification. Methods: Tissue inhibitor of metalloproteinases-2 (TIMP-2), insulin-like growth factorbinding protein 7 (IGFBP7) were measured to a cohort of 719 consecutive patients admitted to Intensive Care Unit (ICU).
\end{abstract}

The primary endpoint was the evaluation of clinical performances of the biomarkers focusing on the probability do develop AKI in the first 7 days. Results: The Kaplan-Meier analysis considering the first 7 days of ICU stay suggested a lower risk of developing AKI $(p<0.0001)$ for patients with a negative (<0.3; TIMP-2*IGFBP7) test. Conclusion: (TIMP$2 *$ IGFBP7) at ICU admission has a good performance in predicting AKI, especially in the first 4 days in ICU.

Video Journal Club 'Cappuccino with Claudio Ronco' at http://www.karger.com/?doi=485591. @ 2018 S. Karger AG, Basel

\section{Background}

Acute Kidney Injury (AKI) is now recognized as a major global public health concern, with an incidence of about 2,100 per million population, leading to increased
KARGER

(C) 2018 S. Karger AG, Basel

E-Mail karger@karger.com

www.karger.com/bpu
Prof. Claudio Ronco

Department of Nephrology, Dialysis and Transplantation

International Renal Research Institute of Vicenza (IRRIV), San Bortolo Hospital

Viale Rodolfi, 37, IT-36100 Vicenza (Italy)

E-Mail cronco@goldnet.it 
morbidity and mortality, with high financial health care costs and huge social impact $[1,2]$. AKI affects up to $50 \%$ of critically ill patients and its development in Intensive Care Unit (ICU) is independently associated with increased ICU length of stay, risk of long-term renal dysfunction (chronic kidney disease and end-stage renal disease) and short- and long-term mortality [3]. AKI is characterised by a rapid (h to days) deterioration of kidney function while clinical consequences include the accumulation of waste products, electrolytes, fluids, and also less obvious effects, including reduced immunity and dysfunction of non-renal organs (organ cross-talk) [4]. Traditionally, AKI diagnosis is based on a rise in serum creatinine and/or fall in urine output. The definition of AKI has evolved from the Risk, Injury, Failure, Loss, Endstage criteria in 2004 to the AKI Network classification in 2007 [5, 6]. In 2012, both were merged resulting in the Kidney Disease Improving Global Outcomes (KDIGO) classification [7].

Serum creatinine (SCr) and urine output are markers of excretory function and do not provide any information about any other role of the kidney, that is, metabolic, endocrine, or immunological functions. Furthermore, there are potential pitfalls in making AKI diagnosis relying only on creatinine and urine criteria. It has been shown that there are patients that fulfill AKI definition but do not have AKI, and there are also patients with clear evidence of renal injury who do not meet the creatinine or urine criteria for AKI $[8,9]$. Over the past decade, the innovative and emerging proteomic technology has enabled the identification of novel biomarkers that allow improved risk stratification, more informed clinical diagnosis and therapeutic decisions.

\section{AKI Biomarkers}

Tissue inhibitor of metalloproteinases-2 (TIMP-2), which inhibits the activity of various metalloproteinases, and insulin-like growth factor-binding protein 7 (IGFBP7) are soluble proteins, expressed in kidney and other tissues, that are involved in the phenomenon of G1 cell cycle arrest following cell injury [10-12]. According to some authors, the G1 cell-cycle arrest following injury could prevent cells from dividing when the deoxyribo nucleic acid is damaged so that the process of cell division is prevented until the damage is repaired $[11,13]$.

Markers of cell-cycle arrest may signal that the renal epithelium has been stressed and any functional activity has been shut down but may still recover without any per- manent injury to the organ [14], if the causative agent ceases. Importantly, both TIMP-2 and IGFBP7 appear to be able to signal in autocrine and paracrine fashions [12, $15,16]$ thus spreading the "alarm" from the site of injury. There is increased release of TIMP-2 and IGFBP7 in response to a variety of insults such as inflammation, oxidative stress, ultraviolet radiation, drugs and toxins [13], and this may help explain their role as marker for the risk of AKI, which is not a single disease but a complex syndrome with multiple underlying etiologies [17].

The NephroCheck ${ }^{\circledR}$ (NC) Test System is a commercially available, accurate immunoassay test that quantitatively measures the urinary concentrations of TIMP-2 and IGFBP-7, and combines them into a single numerical result, the AKIRisk ${ }^{\circledR}$ Score, which provides a quantitative risk index validated in prospective clinical trials by comparison to clinical endpoints.

A 0.3 cutoff of AKIRisk ${ }^{\circledR}$ Score for the NephroCheck ${ }^{\circledR}$ Test has been established to achieve high sensitivity while preserving acceptable specificity $[14,18,19]$. The chosen cutoff allows the clinician to identify the majority of subjects who will manifest moderate or severe AKI within 12 hours. Patients with an AKIRisk ${ }^{\circledR}$ Score $>0.3$ have 7 times the risk for AKI (95\% CI 4-22) compared to those with lower levels [19]

The objective of this study was to identify how useful $\mathrm{NC}$ is in predicting AKI and AKI free in the first 7 days in a cohort of critically ill patients admitted to the ICU.

\section{Material and Methods}

\section{Ethics Statement}

The study was approved by the local Ethical Committee of the San Bortolo Hospital, Vicenza, Italy. The clinical investigation was conducted according to the principles expressed in the Declaration of Helsinki.

\section{Study Design and Participants}

Enrolled patients were adults admitted to ICU from June 1, 2016 to July 31, 2017 in San Bortolo Hospital (Vicenza, Italy). All patients $\geq 18$ years old were included in the study. Patients on chronic dialysis and with a life expectancy less than $24 \mathrm{~h}$ were excluded. The primary endpoint was the evaluation of clinical performances of the NC Test focusing on the ability to predict the renal injury in the first 7 days. The other outcomes included hospital mortality, combination and duration of stay in intensive care.

Paired serum and urine samples for analysis of serum creatinine and urinary (TIMP-2*IGFBP7) were obtained immediately upon enrollment and analyzed. Serum samples for measurement of SCr were collected every day from $24 \mathrm{~h}$ to 7 day after ICU admission and the day the patient discharged from ICU. Clinical date for the study were collected from the hospital records included baseline demographic, prior health history, SCr, lactic acid, urine 
output, mean arterial pressure, Sequential Organ Failure Assessment (SOFA) scores, Simplified Acute Physiology Score II scores.

In addition, the dates of renal replacement therapy (RRT), death, and ICU discharge were collected.

SCr was measured by enzymatic method with an automatic analyzer (Dimension Vista, Siemens healthcare, Tarrytown, NY, USA). Urine samples were analyzed for TIMP-2 and IGFBP7 by technicians blinded to clinical date using $\mathrm{NC}^{\circledR}$ (Astute Medical, San Diego, CA, USA). The ASTUTE140 Meter automatically multiplies the concentrations of the 2 biomarkers together and divides this product by 1,000 to report a single numerical test result with units of $(\mathrm{ng} / \mathrm{mL}) 2 / 1,000$ (the units for all [TIMP-2*IGFBP7] test and cutoff values in this report). According to the optimal predict of AKI, AKIRisk score $>0.3$ was defined as positive $(+)$ and the score $\leq 0.3$ was defined as negative $(-)$. AKI was defined according to the KDIGO consensus criteria [7] as the increase in SCr by $\geq 0.3$ $\mathrm{mg} / \mathrm{dL}$ ( $\geq 26.5 \mathrm{umol} / \mathrm{L}$ ) or increase in SCr to $\geq 1.5$ times baseline, or urine volume $<0.5 \mathrm{~mL} / \mathrm{kg} / \mathrm{h} \times 6 \mathrm{~h}$. Baseline SCr was defined as the concentration obtained at 6-3 months before hospital admission or at ICU admission in critically ill patients when previous values were not available. We used the terms $\mathrm{NC}(+)$ or $\mathrm{NC}(-)$ according to $\mathrm{NC}$ values meaning for optimal AKI prediction and the terms $\mathrm{AKI}(+)$ or $\mathrm{AKI}(-)$ to consensus diagnostic increases in serum creatinine defining AKI. Patients were classified as follows: $1 \mathrm{NC}(+)$ 2. $\mathrm{NC}(-)$

AKI free (AKI-F) was defined as the probability to be Free from Kidney damage evaluated by KDIGO AKI definitions.

\section{Statistical Analysis}

Continuous variables were described as mean \pm SD or median and interquartile range (IQR). Percentages were calculated for categorical variables. Subjects were grouped based on the values of NC, Mann-Whitney test was used for 2-group comparison. Categorical variables were compared between the 2 groups using Fisher exact test or the chi-square test. Finally, all $p$-values were 2 -sided and a $p$-value $<0.05$ was considered statistically significant. Time to kidney injury during ICU stay was modeled as a function of NC test, possibly adjusting by other covariates. Subsequently, optimal cutoff value for $\mathrm{NC}$ test was estimated as that minimizing the distance between PROC plot and point (0.1). Benjamini and Yekutieli [20] adjusted $p$ values were calculated to account for multiplicity of testing (overall 16 tests, one for each variable considered). For sake of completeness, both $p$-values are reported: unadjusted (without multiplicity correction) and with Benjamini and Yekutieli [20] correction. SPSS Statistics version 22.0 (IBM, Chicago, IL, USA) and R 3.4.2 were used for the analysis.

\section{Results}

Seven hundred nineteen patients were enrolled in the study 389 of which had urinary NC test + and 330 a negative test (Fig. 1). The Epidemiological data and laboratory tests related to renal functionality are displayed in Table 1. There was no statistically significant difference regarding age, gender distribution, body mass index and prevalence of hypertension and diabetes mellitus between

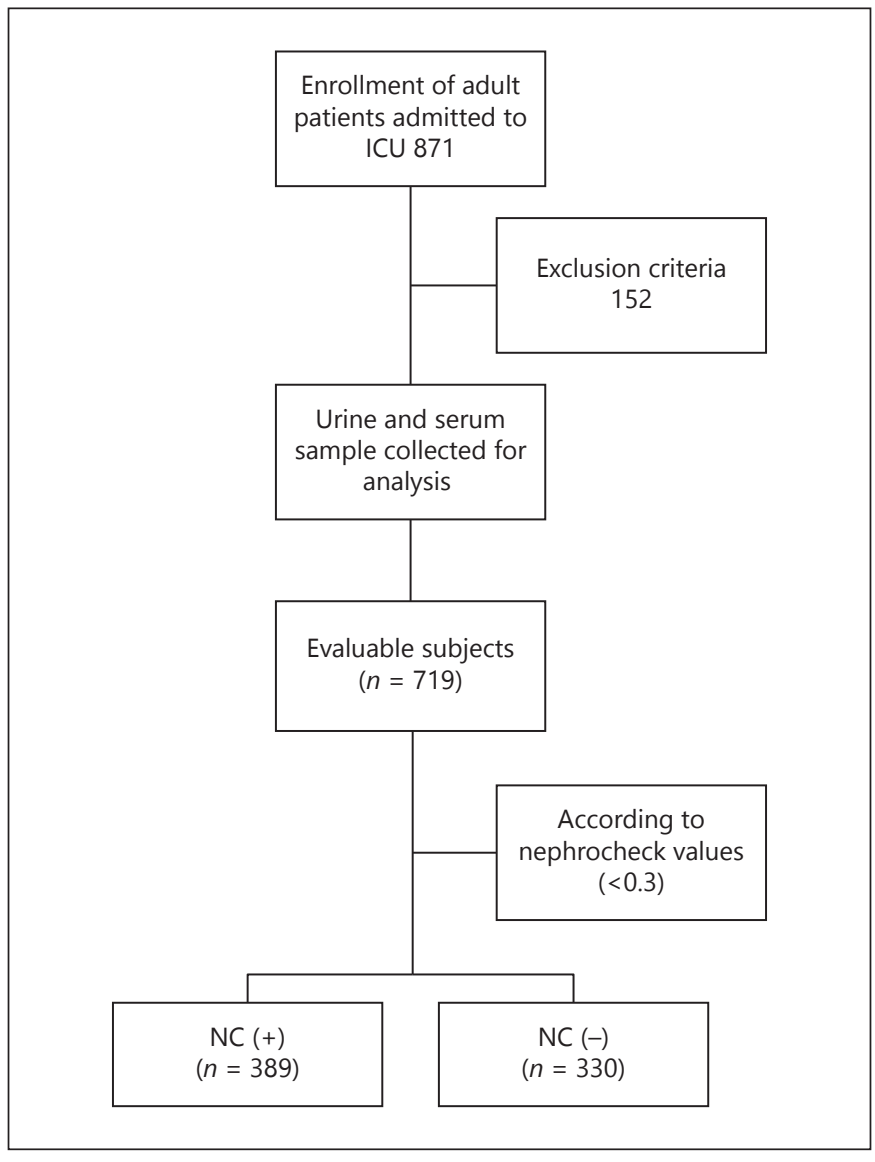

Fig. 1. Study flowchart. AKI free probability.

the 2 groups. The proportion of CKD was significantly higher in the $\mathrm{NC}+$ than the NC-. NC, SAPS II, SOFA, Lactate, and SCr at study enrollment and at ICU discharge were significantly higher in the $\mathrm{NC}+$ compared to the NC-. In addition, the NC+ group displayed significantly lower values for mean arterial pressure compared to the NC- group $(p<0.0001)$. Twenty eight patients $(7.2 \%)$ in the $\mathrm{NC}+$ group received continuous renal replacement therapy (CRRT), 8 patients $(2.4 \%)$ in the NC$(p=0.003)$. The proportion of death in $\mathrm{NC}+$ group was significantly higher than the $\mathrm{NC}$ - group $(p<0.007)$. The length of the ICU stay (LOS) was no significantly difference between 2 groups $(p=0.629)$, but AKI days/ICU days was 2.14-fold higher in the $\mathrm{NC}+$ group compared to the NC- $(p<0.0001)$. The Kaplan-Meier analysis considering the first 7 days of ICU stay suggested a lower risk of developing AKI $(p<0.0001)$ for patients with a negative $\mathrm{NC}$. In NC+ group, the AKI free probability in the first 7 days is $51.9 \%$, while in the $\mathrm{NC}$ - group the AKI free probability rise to $64.2 \%$ (Fig. 2 ). The probability to have an 
Table 1. Characteristics of the study population

\begin{tabular}{|c|c|c|c|c|}
\hline Total $(n=719)$ & $\mathrm{NC}(+)(n=389)$ & $\mathrm{NC}(-)(n=330)$ & $p$ value & $\begin{array}{l}\text { Adjusted } \\
p \text { value* }^{*}\end{array}$ \\
\hline Age, years, median (IQR) & $69.00(52.00-78.00)$ & $68.00(51.25-77.75)$ & 0.542 & 1 \\
\hline BMI, $\mathrm{kg} / \mathrm{m}^{2}$, median (IQR) & $24.98(22.86-28.35)$ & $24.92(22.86-27.74)$ & 0.160 & 0.719 \\
\hline Hypertension, $n(\%)$ & $168(43.2)$ & $162(49.1)$ & 0.113 & 0.550 \\
\hline Diabets mellitus, $n(\%)$ & $61(15.7)$ & $53(16.1)$ & 0.890 & 1 \\
\hline SAPS II, median (IQR) & $42.00(31.75-52.00)$ & $37.50(27.00-50.00)$ & 0.028 & 0.148 \\
\hline SOFA, median (IQR) & $7.00(5.00-10.00)$ & $6.00(3.00-8.00)$ & 0.000 & 0.007 \\
\hline MAP, mm Hg, median (IQR) & $77.00(66.75-90.00)$ & $83.00(71.00-96.00)$ & 0.000 & 0.007 \\
\hline Lactate, $\mathrm{mmol} / \mathrm{L}$, median (IQR) & $2.10(1.40-3.60)$ & $1.60(1.20-2.30)$ & 0.000 & 0.007 \\
\hline SCr admission ICU, mg/dL, median (IQR) & $0.97(0.77-1.48)$ & $0.80(0.63-1.03)$ & 0.000 & 0.007 \\
\hline SCr discharge ICU, mg/dL, median (IQR) & $0.85(0.59-1.24)$ & $0.76(0.60-1.01)$ & 0.000 & 0.007 \\
\hline
\end{tabular}

* Benjamini and Yekutieli [20] adjusted $p$ values calculated to account for multiplicity of testing (overall 16 tests, one for each variable considered). MAP, mean arterial pressure; SCr, serum creatinine; LOS, length of stay; SOFA, Sequential Organ Failure Score; SAPS, Simplified Acute Physiology Score; BMI, body mass index; AKI, Acute Kidney Injury; ICU, Intensive Care Unit; CRRT, Continuous Renal Replacement Therapy.

AKI episode in the first 4 days is $18.7 \%$ higher for patients with a positive $\mathrm{NC}(\mathrm{NC}+41.3 \mathrm{vs}$. NC- $22.6 \%)$. The ability to discriminate patients who will develop AKI decrease in the following days.

The optimal NC cutoff for our population is 0.37 , and it was calculated minimizing the distance between the PROC plot and point (0.1; Fig. 3 ). AUC was 0.633 , Specificity and Sensitivity were 56 and 64\%, respectively, demonstrating that the NC test captured the majority of the AKI (positive) cases and a high number who did not manifest AKI. The number of False Positive was 209 (29\%) with 86 False Negative. The confusion matrix on Table 2 have been developed for both the 0.3 (NC validated cutoff) and the 0.37 as a result of our best cutoff for the prediction of AKI. Critically ill patients with a diagnosis of AKI had significantly higher median urinary (TIMP-2*IGFBP7) AKIRisk of 0.65 (IQR $0.17-3.35$ ) compared with 0.28 (IQR 0.08-0.85) for those without AKI ( $p=0.000$; Fig. 4).

\section{Discussion}

We assumed that specific biomarkers have prognostic value in critically ill patients who subsequently develop AKI and add incremental prognostic information to known clinical risk factors. In this study, our results demonstrate that patients with AKI have higher urinary levels of NC value at ICU admission than those without AKI. Additionally, Urinary levels of NC have independent value in discriminating patients with AKI-F in the first 7 days in ICU. Indeed, patients with lower AKI-F have higher NC value than patients with higher AKI-F.

To date, numerous novel biomarkers of tubular impairment, such as neutrophil gelatinase-associated lipocalin, kidney injury molecule-1, fatty acid-binding protein and interleukin-18, have been tested for early detection of AKI. Recently, some studies have confirmed that TIMP-2 and IGFBP7 are good predictive biomarkers of AKI [21, 22]. In September 2014, the US Food and Drug Administration allowed the so-called NC test (Astute Medical) to be performed as a tool to help the critical care physicians and nephrologists to prevent the development of AKI by an adequate risk assessment and to stratify population for furthers complications. However, only few studies evaluate the efficiency of (TIMP-2*IGFBP7) in predicting AKI outcome, defined as AKI duration, renal recovery, need for RRT and patient mortality [23]. Kashani et al. [14] demonstrated that the risk for major adverse kidney events, including death, RRT or persistent renal dysfunction within 30 days, increased considerably for NC test above 0.3 and 


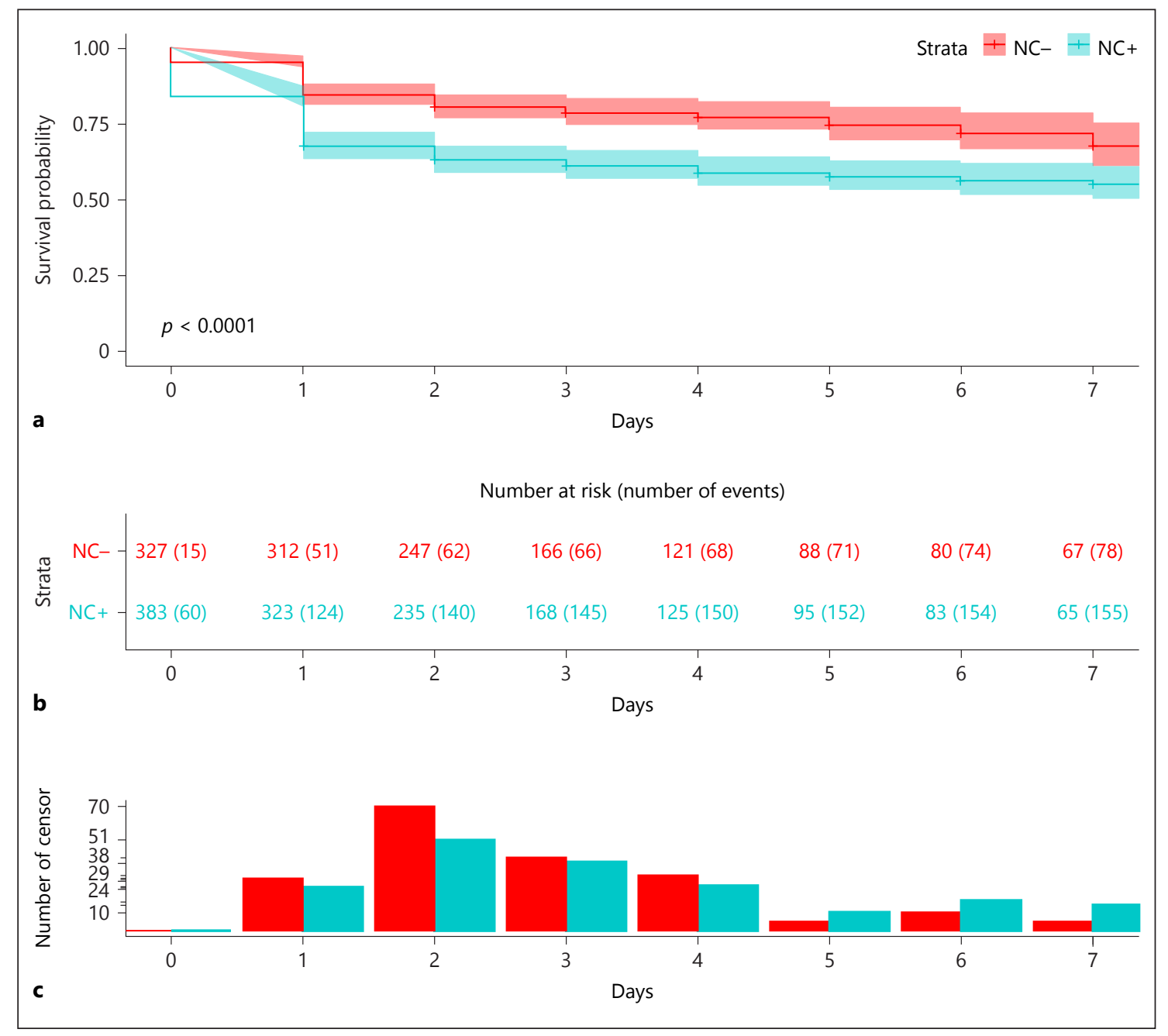

Fig. 2. a The Kaplan-Meier curves describe the time to kidney injury during ICU stay modeled as a function of Nephrocheck test. AKI definition is based on KDIGO AKI stage 1. b The number of patient at risk and the number of AKI events for each day. c Number of censoring events.

doubled when values were $>2.0$. Interestingly, a recently published secondary analysis of the Sapphire study revealed that a single measurement of (TIMP-2*IGFBP7) early in the setting of critical illness is able to identify adult patients with AKI at increased risk for mortality or receipt of RRT over the subsequent 9 months [24]. In a recent published paper, Westhoff et al. [25] demonstrated that an once-only urinary (TIMP-2*IGFBP7) measurement is a good predictor for 30 day and 3 month-mortality (AUC 0.79 and 0.84 , respectively). A lower performance for the prediction of RRT (AUC 0.67) was observed in a heterogeneous neonatal and pediatric cohort of established primary and secondary AKI according to pRIFLE criteria. To our knowledge, no single study has been published so far dem- onstrating the value of urinary levels of $\mathrm{NC}$ in predicting the increased risk of AKI in the first 7 days in ICU patients. Our data show that the value of urinary NC has a moderate prediction of AKI events in the first 7 days in ICU (AUC = 0.633 considering the optimal cutoff 0.37 resulting from our data). Patients with positive urinary levels of NC have lower AKI-F in the first 7 days in ICU than those with negative NC value (51.9 vs. $61.2 \%$ ). The ability to discriminate patients who will develop AKI increase until day 4 . An early identification of patients at risks of developing AKI may help nephrologists and critical care physicians to partially assess the recurrence of the disease which is not a single organ failure dysfunction but a complex syndrome with multiple underlying etiologies. 
Table 2. Confusion matrix for NephroChekc (NC) with 2 different cut off: 0.3 from $\mathrm{NC}$ validation studies and the optimal cut off of 0.37

\begin{tabular}{llll}
\hline & \multicolumn{2}{c}{ AKI status (KDIGO 1, 2, 3) } & $\begin{array}{l}\text { Total number } \\
\text { of tests }\end{array}$ \\
\cline { 2 - 3 } & AKI, $n(\%)$ & No AKI, $n(\%)$ & \\
\hline AKIRISK score $>0.3$ & $158(22)$ & $231(32)$ & 362 \\
AKIRISK score $>0.37$ & $\begin{array}{l}153(21)^{*} \\
\text { TP }\end{array}$ & $\begin{array}{l}209(29)^{*} \\
\text { FP }\end{array}$ & $389^{*}$ \\
\hline AKIRISK score $\leq 0.3$ & $86(12)$ & $271(38)$ & 357 \\
AKIRISK score $\leq 0.37$ & $81(11)^{*}$ & $249(35)^{*}$ & $350^{*}$ \\
\hline Total number of tests & FN & TN & 719 \\
\hline \multirow{2}{*}{$*$ Resulting from the ROC curve of Figure 2.} & 480 & \\
\hline
\end{tabular}

AKI is independently associated with increased ICU length of stay, risk of long-tem renal dysfunction (chronic kidney disease and end-stage renal disease) and shortand long-term mortality [3]. Our data confirm previous findings where a better survival and a lower percentage of CRRT were found for patients with a negative NC [14, 24]. Moreover, the percentage of days with AKI during the ICU stay is the double on patients with a positive NC ( 0.30 vs. 0.14 ). Conversely, we did not find a statistically significant difference on the LOS between the 2 groups. The optimal cut off observed in our population is 0.37 , similar to those validated for the NC test even if we consider patients with AKI at any time and not only in the first $12 \mathrm{~h}$.

This study has several limitations. First, we analyzed 719 ICU patients, but it is a single-center study, the results should require a validation in a multicenter setting, so it lacked power. Second, our study cohort includes all ICU admission patients, not only patients with moderate or high risks patients, as suggested by the studies done for the NC validation. Elevated urinary (TIMP$2 *$ IGFBP7) predicts an increased risk of moderate - severe $A K I$ over the next $12 \mathrm{~h}$; patients with values $>0.3$ have 7 times the risk for AKI (95\% CI 4-22) compared to those with lower levels [19]. Therefore, false positive results will be quite common and will be magnified if the test is inappropriately used in low-risk patients. We found better results in terms of both True Positive 153 (21\%) and False positive 209 (29\%), if compared on the studies involving the (TIMP-2*IGFBP7), but a ten-fold higher values of False Negative. This may depend on a later development of AKI, suggesting a potential utilization of the biomarker also in the days after ICU admission. In this analysis, we included all ICU patients, so the

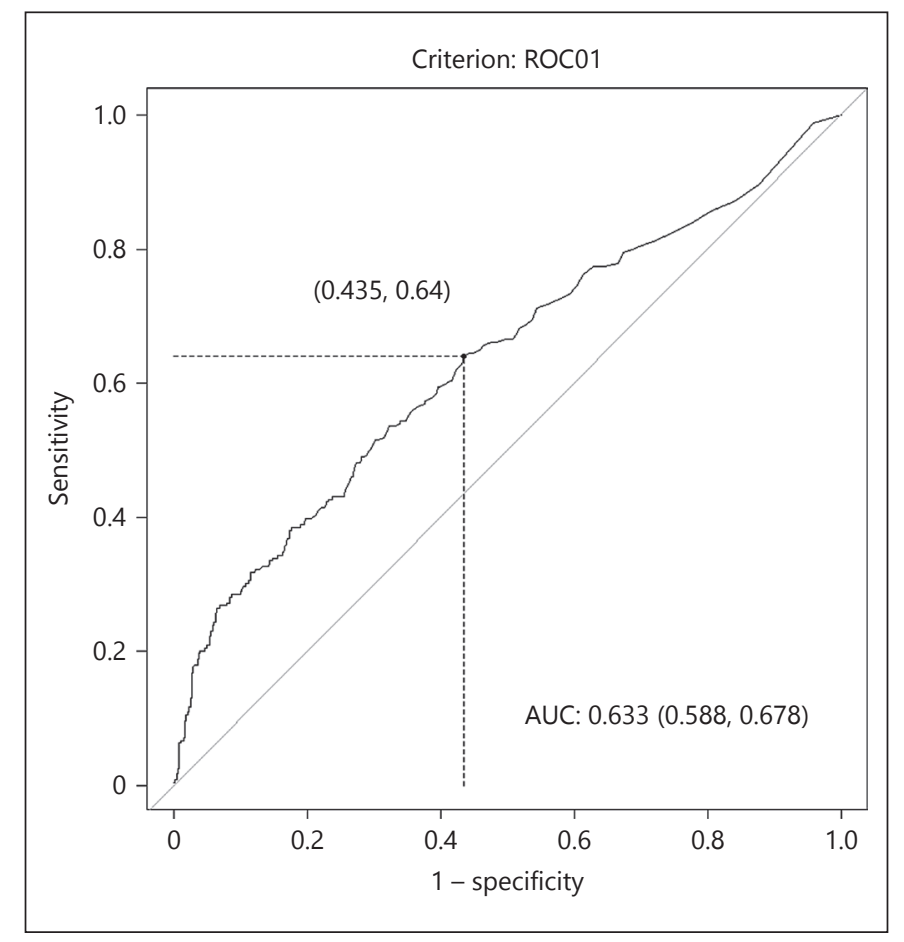

Fig. 3. The PROC curve.

third limitation of our study is the mixture of diagnosis including sepsis, trauma, post-surgery, multi-organ dysfunction, and so on, which comprised different subtypes of AKI. Last but not the least, many patients on ICU admission have already developed AKI: the time interval between onset of AKI and study enrollment was variable. As the respective half-life of the biomarkers is short, we might have missed earlier increases in urinary levels of NC. 
Fig. 4. Box plots of urinary (TIMP2*IGFBP7) AKIRisk score. Subjects with acute kidney injury had significantly higher (TIMP-2*IGFBP7) test values than subjects without AKI ( 0.65 vs. $0.28, p=0.000)$.

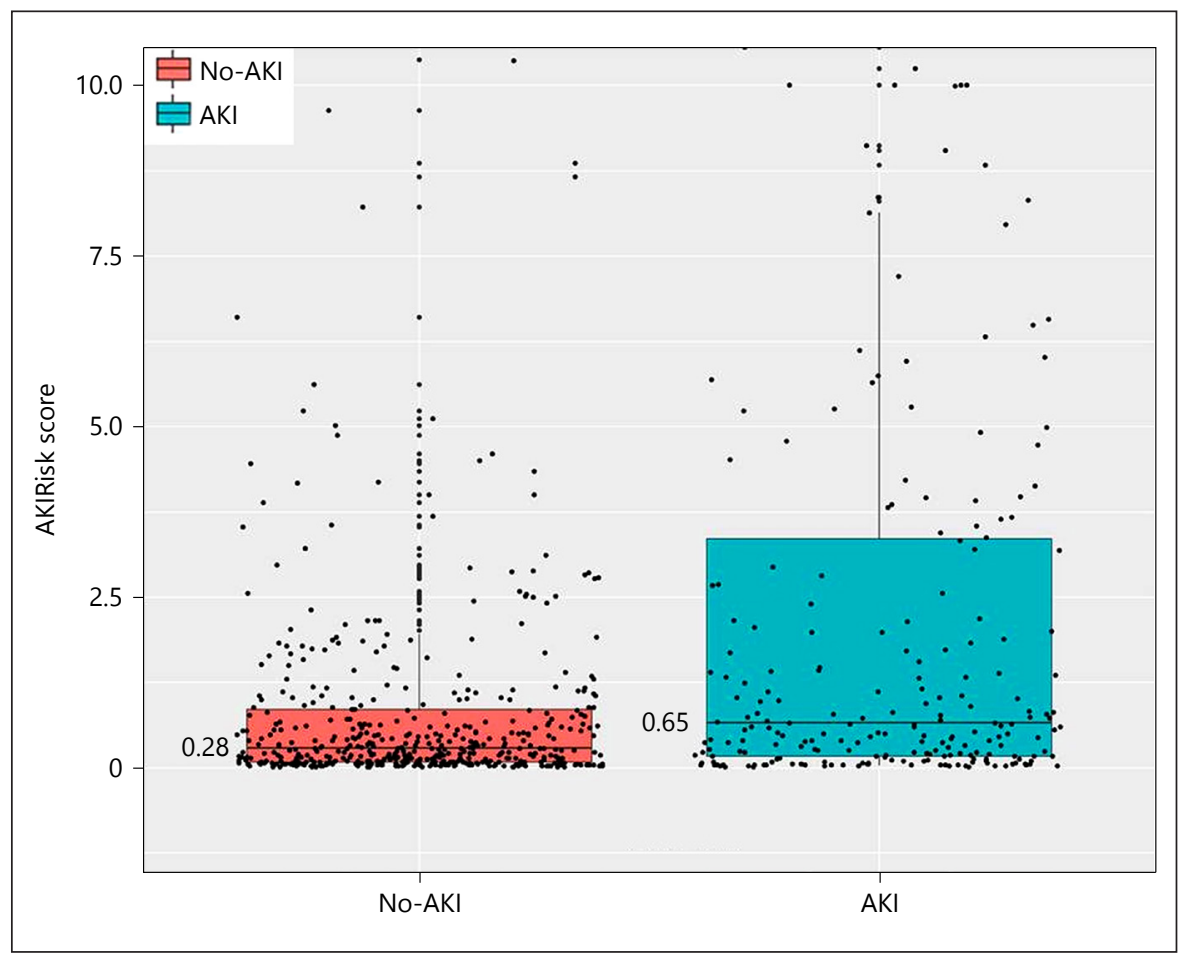

\section{Conclusions}

In conclusion, the nature of the critically ill patient is complex and requires often a careful analysis and a multidisciplinary approach [26-30]. This study suggests that ICU admission measurement of (TIMP-2*IGFBP7) has a good performance in predicting AKI-F in first 4 days in ICU, and a moderate diagnostic performance in predicting AKI in all critically ill patients. There seems to be a less precise predicting capacity of biomarkers when the studied cohort of patients includes several classes of risk. However, the data from this study may help nephrologists and critical care physicians to identify patients at risk for AKI and may affect their clinical behavior utilizing checklists or therapeutic bundles. Further studies are undergoing to confirm and extend our findings with the aim of improving clinical outcome and reducing mortality in ICU patients with AKI although these results have prompted the creation of a nephrology rapid response team that has already demonstrated its utility [31-33]. A correct identification of patients at risk for AKI may allow an earlier intervention and a reduced resource utilization.

\section{Disclosure Statement}

C.R. is consultant of Astute Medical, OCD, Asahi Medical, Baxter, Toray medical. None of the other authors has financial interest related to this study to disclose. No grants have been received for the conduction of this study.

\section{References}

1 Ali T, Khan I, Simpson W, Prescott G, Townend J, Smith W, Macleod A: Incidence and outcomes in acute kidney injury: a comprehensive population-based study. J Am Soc Nephrol 2007;18:1292-1298.

2 Ronco C, Ricci Z: The concept of risk and the value of novel markers of acute kidney injury. Crit Care 2013;17:117.

3 Wang Y, Fang Y, Teng J, Ding X: Acute kidney injury epidemiology: from recognition to intervention. Contrib Nephrol 2016;187:1-8. 4 Ostermann M, Joannidis M: Acute kidney injury 2016: diagnosis and diagnostic workup. Crit Care 2016;20:299.

5 Bellomo R, Ronco C, Kellum JA, Mehta RL, Palevsky P: Acute renal failure - definition, outcome measures, animal models, fluid therapy and information technology needs: the Second International Consensus Conference of the Acute Dialysis Quality Initiative
(ADQI) Group. Crit Care 2004;8:R204-R212. 6 Mehta RL, Kellum JA, Shah SV, Molitoris BA, Ronco C, Warnock DG, Levin A: Acute Kidney Injury Network: report of an initiative to improve outcomes in acute kidney injury. Crit Care 2007;11:R31.

7 Kidney Disease: Improving Global Outcomes (KDIGO) Acute Kidney Injury Work Group: KDIGO clinical practice guideline for acute kidney injury. Kidney Int Suppl 2012;1-138. 
8 Ostermann M: Diagnosis of acute kidney injury: Kidney Disease Improving Global Outcomes criteria and beyond. Curr Opin Crit Care 2014;20:581-587.

9 Thomas ME, Blaine C, Dawnay A, Devonald MA, Ftouh S, Laing C, Latchem S, Lewington A, Milford DV, Ostermann M: The definition of acute kidney injury and its use in practice. Kidney Int 2015;87:62-73.

10 Devarajan P: Update on mechanisms of ischemic acute kidney injury. J Am Soc Nephrol 2006;17:1503-1520.

11 Rodier F, Campisi J, Bhaumik D: Two faces of p53: aging and tumor suppression. Nucleic Acids Res 2007;35:7475-7484.

12 Seo DW, Li H, Qu CK, Oh J, Kim YS, Diaz T, Wei B, Han JW, Stetler-Stevenson WG: Shp-1 mediates the antiproliferative activity of tissue inhibitor of metalloproteinase- 2 in human microvascular endothelial cells. J Biol Chem 2006;281:3711-3721.

13 Price PM, Safirstein RL, Megyesi J: The cell cycle and acute kidney injury. Kidney Int 2009;76:604-613.

14 Kashani K, Al-Khafaji A, Ardiles T, Artigas A, Bagshaw SM, Bell M, Bihorac A, Birkhahn R, Cely CM, Chawla LS, Davison DL, Feldkamp T, Forni LG, Gong MN, Gunnerson KJ, Haase M, Hackett J, Honore PM, Hoste EA, JoannesBoyau O, Joannidis M, Kim P, Koyner JL, Laskowitz DT, Lissauer ME, Marx G, McCullough PA, Mullaney S, Ostermann M, Rimmele T, Shapiro NI, Shaw AD, Shi J, Sprague AM, Vincent JL, Vinsonneau C, Wagner L, Walker MG, Wilkerson RG, Zacharowski K, Kellum JA: Discovery and validation of cell cycle arrest biomarkers in human acute kidney injury. Crit Care 2013;17:R25.

15 Seo DW, Li H, Guedez L, Wingfield PT, Diaz T, Salloum R, Wei BY, Stetler-Stevenson WG: TIMP-2 mediated inhibition of angiogenesis: an MMP-independent mechanism. Cell 2003; 114:171-180.

16 Stetler-Stevenson WG: Tissue inhibitors of metalloproteinases in cell signaling: metalloproteinase-independent biological activities. Sci Signal 2008;1:re6.
17 Chawla LS, Amdur RL, Amodeo S, Kimmel PL, Palant CE: The severity of acute kidney injury predicts progression to chronic kidney disease. Kidney Int 2011;79:1361-1369.

18 Hoste EA, McCullough PA, Kashani K, Chawla LS, Joannidis M, Shaw AD, Feldkamp T, Uettwiller-Geiger DL, McCarthy P, Shi J, Walker MG, Kellum JA; Sapphire Investigators: Derivation and validation of cutoffs for clinical use of cell cycle arrest biomarkers. Nephrol Dial Transplant 2014;29: 2054-2061.

19 Bihorac A, Chawla LS, Shaw AD, Al-Khafaji A, Davison DL, Demuth GE, Fitzgerald R, Gong MN, Graham DD, Gunnerson K, Heung M, Jortani S, Kleerup E, Koyner JL, Krell K, Letourneau J, Lissauer M, Miner J, Nguyen HB, Ortega LM, Self WH, Sellman R, Shi J, Straseski J, Szalados JE, Wilber ST, Walker MG, Wilson J, Wunderink R, Zimmerman J, Kellum JA: Validation of cell-cycle arrest biomarkers for acute kidney injury using clinical adjudication. Am J Respir Crit Care Med 2014;189:932-939.

20 Benjamini Y, Yekutieli D: The control of the false discovery rate in multiple testing under dependency. Ann Stat 2001;29:1165-1188.

21 Wetz AJ, Richardt EM, Wand S, Kunze N, Schotola H, Quintel M, Brauer A, Moerer O: Quantification of urinary TIMP-2 and IGFBP-7: an adequate diagnostic test to predict acute kidney injury after cardiac surgery? Crit Care 2015;19:3.

22 Yamashita T, Doi K, Hamasaki Y, Matsubara T, Ishii T, Yahagi N, Nangaku M, Noiri E: Evaluation of urinary tissue inhibitor of metalloproteinase- 2 in acute kidney injury: a prospective observational study. Crit Care 2014; 18:716.

23 Koraishy FM, Coca SG: Can we predict recovery from severe acute kidney injury with biomarkers? Semin Dial 2014;27:236-239.

24 Koyner JL, Shaw AD, Chawla LS, Hoste EA, Bihorac A, Kashani K, Haase M, Shi J, Kellum JA; Sapphire Investigators: Tissue inhibitor metalloproteinase-2 (TIMP-2) IGF-binding protein-7 (IGFBP7) levels are associated with adverse long-term outcomes in patients with AKI. J Am Soc Nephrol 2015;26:17471754.

25 Westhoff JH, Tonshoff B, Waldherr S, Poschl J, Teufel U, Westhoff TH, Fichtner A: Urinary Tissue Inhibitor of Metalloproteinase-2 (TIMP-2) Insulin-like growth factor-binding protein 7 (IGFBP7) predicts adverse outcome in pediatric acute kidney injury. PLoS One 2015;10:e0143628.

26 Ronco C, Brendolan A, Dan M, Piccinni P, Bellomo R, De Nitti C: Adsorption in sepsis. Kidney Int 2000;58:S148-S155.

27 Camussi G, Ronco C, Montrucchio G, Piccoli G: Role of soluble mediators in sepsis and renal failure. Kidney Int Suppl 1998;66:S38S42.

28 Ronco C, Brendolan A, Crepaldi C, Rodighiero M, Scabardi M: Blood and dialysate flow distributions in hollow-fiber hemodialyzers analyzed by computerized helical scanning technique. J Am Soc Nephrol 2002;13(suppl 1):S53-S61.

29 Ronco C, Di Lullo L: Cardiorenal syndrome. Heart Fail Clin 2014;10:251-280.

30 Bellomo R, Ronco C: Blood purification in the intensive care unit: evolving concepts. World J Surg 2001;25:677-683

31 Rizo-Topete LM, Rosner MH, Ronco C: Acute kidney injury risk assessment and the nephrology rapid response team. Blood Purif 2017;43:82-88

32 Levante C, Ferrari F, Manenti C, Husain-Syed F, Scarpa M, Hinna Danesi T, De Cal M, Corradi $V$, Virzì GM, Brendolan A, Nalesso F, Bezerra P, Lopez-Giacoman S, Samoni S, Senzolo M, Giavarina D, Salvador L, Bonato R, De Rosa S, Rettore E, Ronco C: Routine adoption of TIMP2 and IGFBP7 biomarkers in cardiac surgery for early identification of acute kidney injury. Int J Artif Organs 2017, Epub ahead of print.

33 Ronco C, Rizo-Topete L, Serrano-Soto M, Kashani K: Pro: prevention of acute kidney injury: time for teamwork and new biomarkers. Nephrol Dial Transplant 2017;32:408413 